\title{
Detection of $A P C$ gene deletion by double competitive polymerase chain reaction in patients with familial adenomatous polyposis
}

\author{
MAHO TAKAHASHI ${ }^{1,3}$, MARIKO KIKUCHI $^{1,3}$, NAGANARI OHKURA $^{1}$, HIROKO YAGUCHI $^{1}$, \\ YUKO NAGAMURA $^{1}$, SUMIKO OHNAMI $^{2}$, MINEKO USHIAMA ${ }^{2}$, TERUHIKO YOSHIDA ${ }^{2}$, \\ KOKICHI SUGANO ${ }^{4}$, TAKEO IWAMA ${ }^{5}$, SHINJI KOSUGI ${ }^{6}$ and TOSHIHIKO TSUKADA ${ }^{1}$
}

\begin{abstract}
${ }^{1}$ Tumor Endocrinology Project and ${ }^{2}$ Genetics Division, National Cancer Center Research Institute, 5-1-1 Tsukiji, Chuo-ku, Tokyo 104-0045; ${ }^{3}$ Department of Ophthalmology, Teikyo University School of Medicine, 2-11-1 Kaga, Itabashi-ku, Tokyo 173-8605; ${ }^{4}$ Oncogene and Cancer Prevention Unit, Tochigi Cancer Center Research Institute, 4-9-13 Younan, Utsunomiya, Tochigi 320-0834; ${ }^{5}$ Kyoundo Hospital, Sasaki Institute, 2-2 Kandasurugadai, Chiyoda-ku, Tokyo 101-0062; ${ }^{6}$ Departments of Clinical Genetics and Medical Ethics, Kyoto University Graduate School of Medicine, 54 Shogoinkawahara-cho, Sakyo-ku, Kyoto 606-8397, Japan
\end{abstract}

Received February 3, 2006; Accepted April 19, 2006

\begin{abstract}
Familial adenomatous polyposis (FAP) is an autosomal dominant familial cancer syndrome caused by germline mutations of the tumor suppressor adenomatous polyposis coli $(A P C)$ gene. Heterozygous $A P C$ mutations have been identified in the majority of classical FAP patients who develop more than 100 colorectal adenomas. However, classical FAP patients often fail to display germline $A P C$ mutations detectable by routine mutation analysis. These apparently mutation-negative cases may be caused by heterozygous large genomic deletions. In the present study, FAP patients who showed no APC germline mutation detectable by the protein truncation assay and direct sequencing of protein coding exons were screened for $A P C$ gene deletion by a gene dose assay based on double competitive polymerase chain reaction. Gene dosage measurements within exon 15 of the $A P C$ gene identified two patients with gene deletion and one with possible gene duplication among 41 apparently mutation-negative patients. The deleted sequences in the two patients were determined by fine gene dose mapping around the $A P C$ gene and nucleotide sequencing of the deletion breakpoints. They were $\sim 435$-kilobase pair $(\mathrm{kb})$ and $737-\mathrm{kb}$ regions including the whole $A P C$ gene and flanked by a 4-base pair repeat and LINE-1 repetitive sequences, respectively. The chimeric LINE-1 element created at the breakpoint in the latter case also contained a short sequence derived from
\end{abstract}

Correspondence to: Dr Toshihiko Tsukada, Tumor Endocrinology Project, National Cancer Center Research Institute, 5-1-1 Tsukiji, Chuo-ku, Tokyo 104-0045, Japan

E-mail: ttsukada@gan2.res.ncc.go.jp

Key words: APC, familial adenomatous polyposis, deletion, LINE-1, gene dosage another LINE-1 element, suggesting a complex unequal homologous recombination event. These findings indicate that this gene dose assay is a useful technique to detect large gene deletions of the $A P C$ gene and to determine their genomic breakpoints.

\section{Introduction}

Familial adenomatous polyposis (FAP) (MIM\# 175100) is an autosomal dominant familial cancer syndrome characterized by an early onset of multiple adenomatous polyps of the colon that can progress to malignant forms (1). This disorder is caused by germline mutations of the adenomatous polyposis coli $(A P C)$ gene, the 15 protein-coding exons of which are distributed along a 100-kilobase pair $(\mathrm{kb})$ region on chromosome $5 \mathrm{q} 21$ and encode $\sim 10 \mathrm{~kb}$ mRNA that is translated into a tumor suppressor protein of 2843 amino acids $(2,3)$. Various heterozygous germline mutations of the APC gene have been identified in the majority of classical FAP patients who develop more than 100 colorectal adenomas and early-onset colorectal carcinoma as well as other features including retinal pigment epithelial hypertrophy. However, 20-50\% of classical FAP patients displayed no detectable germline mutations when examined by polymerase chain reaction (PCR)-based analytical methods such as single strand conformational analysis, protein truncation assay (PTT) and direct DNA sequencing of PCR-amplified exons (4-9). Although these mutation-negative cases may represent disease entities distinct from that caused by $A P C$ gene mutation, it is also likely that routine analysis may have missed mutations in the untested part of the $A P C$ gene or large genomic deletions $(6,10)$.

In the routine PCR-based mutation screening, genomic deletions are often masked by the presence of the normal allele in the heterozygous state. Several different analytical techniques have been developed to detect heterozygous gene deletions and applied to DNA diagnosis of familial cancer syndromes (11). We previously demonstrated that a gene 
A

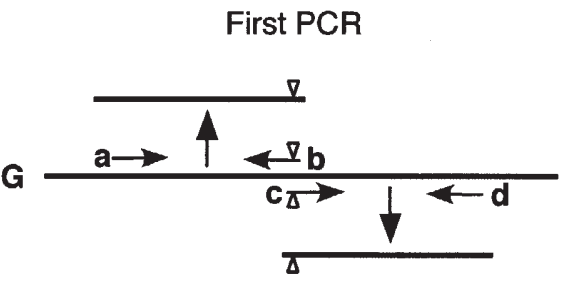

B

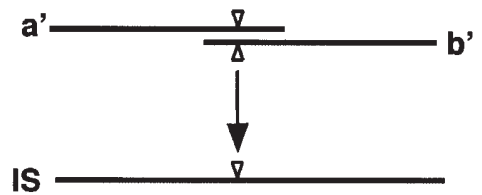

C

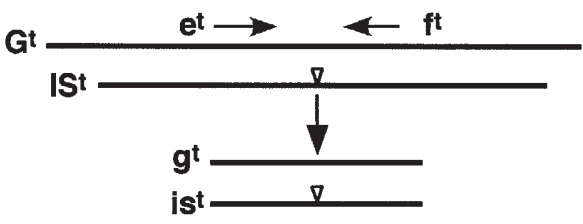

D

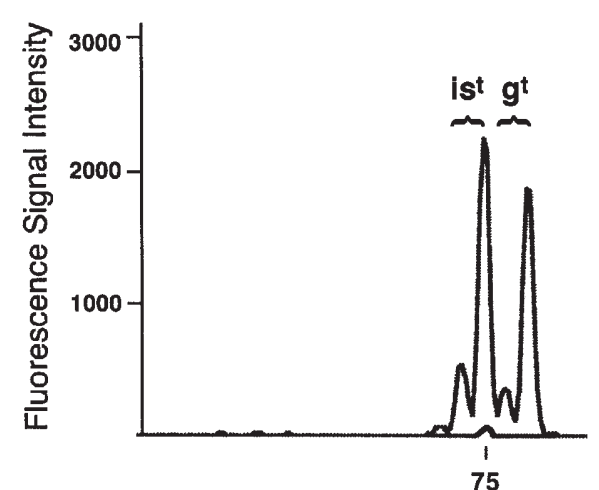

Second PCR

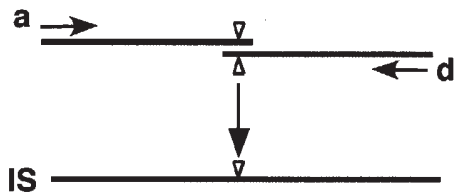

IS
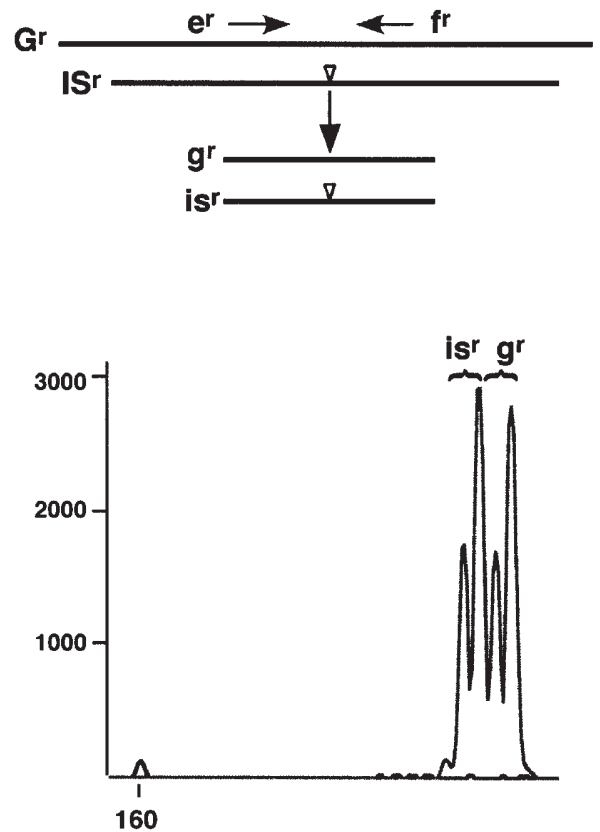

Fragment Size (nucleotide)

Figure 1. Gene dose assay. A, construction of internal standards by the original method. In the first PCR, two half portions of the internal standard were produced with primer pairs a/b or c/d and with genomic DNA $(\mathrm{G})$ as a template. Primers $\mathrm{b}$ and $\mathrm{c}$ had 2-bp deletion (shown by a triangle) in otherwise normal sequences and were complementary to each other within their overlapping sequence. In the second PCR, a full-length internal standard (IS) was generated by extension and amplification of the two half portions with primers a and d. Horizontal arrows and solid lines represent PCR primers and DNA sequences, respectively. B, construction of internal standards by the modified method. Shorter internal standards were produced by extension of two synthetic primers a' and $\mathrm{b}^{\prime}$ that were designed to have short complementary sequences in the 3' ends, where 2-bp deletion was introduced. C, in the gene dose assay, genomic DNA $\left(\mathrm{G}^{\mathrm{t}}\right)$ and internal standard (ISt) sequences for a test region were amplified with primers $\mathrm{e}^{\mathrm{t}}$ and $\mathrm{f}^{\mathrm{t}}$ (left), and genomic DNA $\left(\mathrm{G}^{\mathrm{r}}\right)$ and internal standard (IS ${ }^{\mathrm{r}}$ ) sequences for the reference gene were amplified with primers $\mathrm{e}^{\mathrm{r}}$ and $\mathrm{f}^{\mathrm{r}}$ (right). The PCR products of internal standards is ${ }^{\mathrm{t}}$ and is ${ }^{\mathrm{r}}$ were $2 \mathrm{bp}$ smaller than those of genomic sequences $\mathrm{g}^{\mathrm{t}}$ and $\mathrm{g}^{\mathrm{r}}$, respectively. D, representative electropherogram of PCR products from test genomic $\left(\mathrm{g}^{\mathrm{t}}\right)$ and internal standard (is ${ }^{\mathrm{t}}$ ) sequences (left) and the reference genomic $\left(\mathrm{g}^{\mathrm{r}}\right)$ and internal standard $\left(\mathrm{is}^{\mathrm{r}}\right)$ sequences (right). Each PCR product generated two fluorescent peaks representing $3^{\prime}$-end deoxyadenylated and non-deoxyadenylated forms. Nucleotide numbers below were derived from the GeneScan-500 TAMRA size marker. Gene dose quotient was calculated by the following formula: Gene dose quotient $\left.=\left(\mathrm{g}^{\mathrm{t}} / \mathrm{is}\right)^{\mathrm{t}}\right) /\left(\mathrm{g}^{\mathrm{r}} / \mathrm{is}^{\mathrm{r}}\right)$.

dose assay based on double competitive PCR was useful to determine a large germline deletion of the MEN1 gene in a family with multiple endocrine neoplasia type $1(12,13)$. We partly modified this technique and applied it to detect germline $A P C$ gene deletion in apparently mutation-negative FAP patients. We here demonstrate that this method is useful to identify large genomic deletion and genomic breakpoints in the $A P C$ gene region.

\section{Materials and methods}

Subjects. Patients were diagnosed as having colorectal polyposis by endoscopic examinations at individual hospitals and registered in a multi-institutional research program for hereditary colorectal cancer. Written informed consent was obtained for the APC gene mutation analysis, which was conducted at the National Cancer Center Research Institute. 
Forty-one apparently unrelated colorectal polyposis patients who had no germline APC gene mutation detectable by PTT (14) and DNA sequencing (15) of the whole protein-coding exons were studied. This study was approved by the Ethics Review Committees of the National Cancer Center, Tochigi Cancer Center, Kyoundo Hospital and Kyoto University Graduate School of Medicine.

Construction of internal standard DNA. The gene dose assay was based on quantitative PCR and employed internal standard DNA for competitive PCR, which had a 2-base pair (bp) deletion within otherwise normal sequences. The internal standards for the target sequence within exon 15 of the $A P C$ gene (Ex15) and some other target sequences around the gene and that for the $h M L H 1$ gene, which was used as a reference sequence, were prepared as described previously (13). Briefly, the internal standards were produced by two-step amplification (Fig. 1A and Table I), and introduced into a plasmid vector. The plasmid clones verified to have a desired sequence were selected and cut with restriction enzymes that cleaved only the vector DNA. This linearization of internal standard DNA was essential for high reproducibility of this gene dose assay. This original method provided relatively long internal standard DNA (>300 bp).

In the present study, we additionally adopted a modified method for internal standard preparation, which provided relatively short internal standard DNA $(\sim 100 \mathrm{bp})$. In this new method, synthetic PCR primers (Fig. 1B, Table I) were designed to have a short complementary sequence in their 3'-ends, where 2-bp deletion was introduced into an otherwise normal sequence, and were simply extended by PCR under the following conditions: $1 \mathrm{~min}$ at $95^{\circ} \mathrm{C}$ for 1 cycle; $1 \mathrm{~min}$ at $95^{\circ} \mathrm{C}$ for denaturing, $1 \mathrm{~min}$ at $51,55,59$ or $63^{\circ} \mathrm{C}$ for annealing, $1 \mathrm{~min}$ at $72^{\circ} \mathrm{C}$ for extension for 12 cycles, $5 \mathrm{~min}$ at $72^{\circ} \mathrm{C}$ for 1 cycle in the reaction mixture containing Taq DNA polymerase (rTaq, Takara, Japan), primers (50 $\mu \mathrm{M}$ each) and other components recommended by the enzyme supplier. The PCR products, purified with QIAquick PCR purification kit (Qiagen Inc., Valencia, CA), were diluted to $1: 10^{3}-10^{9}$ and used as internal standard DNA.

The genomic sequence data were obtained from internet database (http://www.ncbi.nlm.nih.gov/). The accession numbers of a chromosome 5q clone CTC-554D6 containing the $A P C$ gene and $3 q$ clones RP11-129K12 and RP11-491D6 containing the $h M L H 1$ gene were AC008575.7, AC011816.17 and AC006583.31, respectively.

Gene dose assay. Genomic DNA was prepared either directly from whole blood with a QIAamp blood kit (Qiagen Inc.) or from isolated peripheral blood cells by the ordinary phenolchloroform method or a SepaGene Kit (Sanko Junyaku, Tokyo, Japan). Double competitive PCR was described previously (Fig. 1C) (13). Some PCR primers for a test or the reference sequence are shown in Table I. One of the primers of each primer pair was labeled with fluorescein. Each sample was amplified in triplicate. The relative amounts of PCR products derived from the genomic and internal standard DNA in each reaction tube were evaluated by measuring fluorescence intensity with an automated capillary sequencer as described previously (Fig. 1D) (13).
Gene dose quotient was calculated from the amounts of four PCR products: Gene dose quotient $=(\mathrm{PCR}$ product of test genomic sequence $\div$ PCR product of internal standard for test sequence $) \div($ PCR product of reference genomic sequence $\div$ PCR product of internal standard for reference sequence). The mean value obtained from triplicate PCR was used to calculate each gene dose quotient. Gene dose was normalized to the normal control DNA by using the following formula: Gene dose $=$ (gene dose quotient of test sample $\div$ gene dose quotient of normal control sample) x 2 .

Microsatellite analysis. Genomic DNA was amplified by PCR with a pair of primers, one of which was labeled with fluorescein. The size of the PCR product was estimated by electrophoresis with an automated capillary sequencer as described previously (12). The primer sequences and PCR conditions were obtained from the Genome Database (http://gdbwww.gdb.org/).

Sequencing analysis of deletion breakpoints. In one patient (KT25) having a gene deletion, PCR primers were prepared at an $\sim 1-\mathrm{kb}$ interval within the region where the deletion breakpoints were located by the gene dose mapping (Fig. 4A). Genomic DNA of the patient or a normal volunteer was amplified by PCR with every possible combination of upstream and downstream primers under the following conditions; $1 \mathrm{~min}$ at $95^{\circ} \mathrm{C}$ for 1 cycle; $30 \mathrm{sec}$ at $95^{\circ} \mathrm{C}, 3 \mathrm{~min}$ at $68^{\circ} \mathrm{C}$ for 35 cycles; $3 \mathrm{~min}$ at $68^{\circ} \mathrm{C}$ for 1 cycle. PCR products were separated by electrophoresis on $1 \%$ agarose gel, and visualized by ethidium bromide staining. PCR products specific to the patient were analyzed by direct nucleotide sequencing with primers used for PCR.

In another patient (KT64), the deletion breakpoints were considered to be within long repetitive sequences. Therefore, long-range PCR was conducted with primers used for gene dose assay at sites where both alleles have proven to be retained. Long-range PCR was performed in a $50 \mu 1$ reaction mixture containing 0.5 unit Takara LA Taq (Takara, Japan), $25 \mathrm{ng}$ genomic DNA, $50 \mu \mathrm{M}$ each primer and components recommended by the enzyme supplier under the following conditions: $1 \mathrm{~min}$ at $94^{\circ} \mathrm{C}$ for $1 \mathrm{cycle}, 10 \mathrm{sec}$ at $98^{\circ} \mathrm{C}$ and $15 \mathrm{~min}$ at $68^{\circ} \mathrm{C}$ for 30 cycles, and extension of $72^{\circ} \mathrm{C}$ for $10 \mathrm{~min}$. PCR products were separated by electrophoresis on $0.8 \%$ agarose gel, and visualized by ethidium bromide staining. PCR products specific to the patient were subjected to restriction enzyme analysis. The restriction fragments considered to have the boundary sequences were introduced into a plasmid vector pBluescriptSK(+) (Stratagene, La Jolla, CA) and analyzed by nucleotide sequencing.

\section{Results}

Gene dose analysis of apparently mutation-negative FAP patients. Gene dose at the test sequence Ex15, which was located near the 5'-end of exon 15 of the $A P C$ gene, was measured in 6 normal subjects and 41 apparently mutationnegative polyposis patients. All normal subjects and all but three polyposis patients displayed gene dose values distributed around 2, suggesting that this group represents subjects having two copies of the Ex15 sequence and that deviation of each 
Table I. PCR primers for preparing internal standards and for gene dose assay.

\section{$\operatorname{Ex} 15$}

a: (127178) CGAAGTACAAGGATGCCAAT

b: (127401) ATTATCATCATGTATTGGTGTC

c: (127380) CACCAATACATGATGATAATAG

d: (127624) CAGTGGTGGAGATCTGCAA

e: (127319) AGGCATCTCATCGTAGTAAGCAGA

f: (127486) TTGATGAAGAGGAGCTGGGTAACA

4

a': (118785) GGAGATGGTGGCTGTCAGCTTGCTAAGCAAGTCTCTTCCCAGCAAGGGCAAGGGAGTCA

b': (118894) CCTGCTCGGTGGGTGGACAAGAAATAGTTCACCAGTTCTTGTACATGCCTGACTCCCTT

e: (118793) TGGCTGTCAGCTTGCTAAGCAAGT

f: (118890) CTCGGTGGGTGGACAAGAAATAGT

5

a': (112608) CTTACCCTGTTTTCAGTGGATCAAGTTTGAAATGTTGTCTCTTTTGTGTTTTGATCAGA

b': (112717) CATCCTGTCGGCCATGATCTCACACTACTTAAGATTTTAAATTAGAACTTCTGATCAAA

e: (112614) CTGTTTTCAGTGGATCAAGTTTGAA

f: (112710) TCGGCCATGATCTCACACTACTTA

13

a': (100961) CTCTTTCTGCAGTGCCTCAACTCTGCTCAGAGGAAGATAAAGCTTCGCATCATTTCAGA

b': (101071) TCCAAAACTATTACAGACAGAAGAGTTTGAGACAATTGAAGGTAAACAGCTCTGAAATG

e: (100964) TTTCTGCAGTGCCTCAACTCTGCT

14

a': (107021) AGAGGTGACATTTAACCCATAACATAAAGGTGAAAAGGAGGGGATCATGGAGGGGTGC

b': (107130) TCAAATACCCCAAGCTCTTTCCAAATACCATTCCCACTGCCTGGAATGTTGCACCCCTT

e: (107036) CCCATACATAAAGGTGAAAAGGAG

f: (107125) TACCCCAAGCTCTTTCCAAATACC

18

a': (115585) TATTCAGTGCTCATGGATTGAAAGACTAAATATTAAGATGTCAATACTACCTAATGCTC

b': (115694) CTGTCTCTGCAAAAACTGTTGTTGGAGTAGGGATTGCATTGCATCTATAGAGCATTAGG

e: (115589) CAGTGCTCATGGATTGAAAGACTA

f: (115690) CTCTGCAAAAACTGTTGTTGGAGT

19

a': (108741) GAGTCTGCTATTGGTCCAGCACACTAAAAATCTCTTAAAGTGACTATATGAAAATGGAA

b': (108852) ATCCAGCCAGAACCTGAGTTTCTTTAACTTATTATTATATCCTACCCATTTTTCCATTT

e: (108742) AGTCTGCTATTGGTCCAGCACACT

28

a': (100778) TCTGATTATAGATTGTGGGTGACATTGTAGGTGGTTGGAGTTAAGAATTACTCCAAAAA

b': (100887) AGATAAGGTAAAAAGCATGCATTCTCTCTAAGTTTCAGGAGCGATTTTTTTTTTGGAGT

e: (100785) ATAGATTGTGGGTGACATTGTAGG

29

a': (94246) CTGAAGCCTCTATCAAATAGAAGGGAAAAAAGAAGTAGGCCTTAGACAAATCCAATGTA

b': (94356) TGCAGTCATTTCCTCCCTCATGTCTTTTTTGGAGATACAGCATCTAAAGCTACATTGGA

e: (94248) GAAGCCTCTATCAAATAGAAGGGA

f: (94354) CAGTCATTTCCTCCCTCATGTCTT

Primers used for gene dosage analysis within exon 15 of the APC gene (Ex5), within the hMLH1 gene (hMLH1), and target sites surrounding the deletion breakpoints $(4,5,13,14,18,19,28$ and 29 shown in Fig. 3) are listed. Primers a, a', b, b', c and d are for internal standard construction and primers e and $\mathrm{f}$ are for gene dose assay (Fig. 1). Primer sequences are shown from 5' (left) to 3 ' (right). Primer positions are indicated in the parenthesis by the $5^{\prime}$ end position numbered in the clone CTC-554D6 (AC008575.7) for APC, in the clone RP11-491D6 (AC006583.31) for $h M L H 1$, in the clone RP11-159K7 (AC137549.1) for 4 and 5, in the clone CTC-487M23 (AC008536.7) for 13 and 14, in the clone RP11-56P2 (AC136500.1) for 18 and 19, and in the clone CTD-2201G3 (AC010431.7) for 28 and 29.

value from 2 in this group should represent an experimental error (Fig. 2). Two patients (KT25 and KT64) showed gene dose values around 1, suggesting that they had only one copy of the Ex15 sequence. Another patient reproducibly displayed a gene dose of $\sim 3$, suggesting the presence of three copies of the Ex15 sequence. Gene dose values of these three patients deviated from 2 by more than $2 \mathrm{x}$ standard deviation calculated from all samples (standard deviation $=0.384, \mathrm{n}=47$ ). 


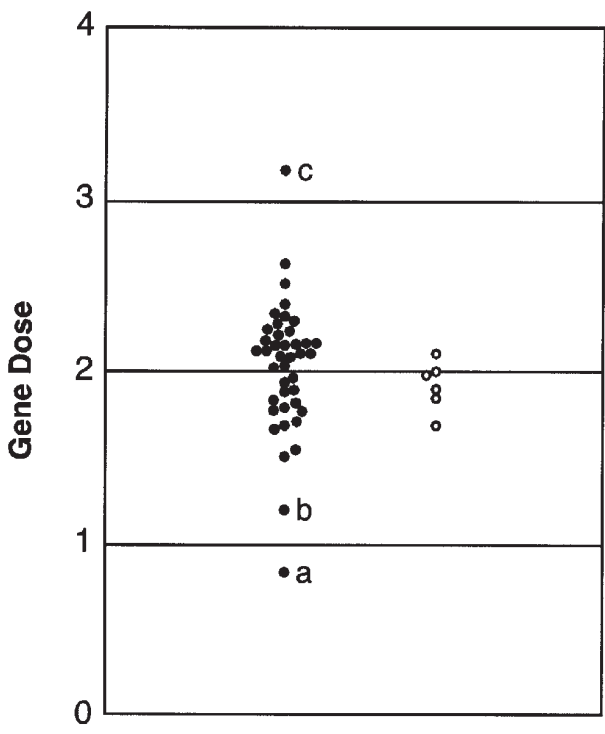

Figure 2. Distribution of gene dose values calculated for 41 polyposis patients (closed circle) and 6 healthy control subjects (open circles). KT25 and KT64 patients are indicated by a and $b$, respectively. A patient with possible gene duplication is indicated by $\mathrm{c}$.

Gene dose mapping in patients with only one copy of the Ex15 sequence. The sequence copy number at multiple sites around the $A P C$ gene was determined by gene dose assay and microsatellite polymorphism analysis in patients KT25 and KT64. The gene dose at several target sites around the gene was measured, and then the next set of target sites was selected within the regions where the genomic breakpoints were found to be located by the first gene dose assay. These procedures were repeated until the breakpoints were located within at most $10-\mathrm{kb}$ regions.

In KT25, the copy number was examined at sites 1-15 and was a half of the normal value at sites 5-13 (Fig. 3), suggesting that the breakpoints were within a $6.2-\mathrm{kb}$ region between sites 4 and 5 and within a 6.1-kb region between sites 13 and 14. In KT64, the copy number was examined at sites 16-31 and was a half of the normal value at sites 19-28 (Fig. 3), suggesting that the breakpoints were within a $6.9-\mathrm{kb}$ region between sites 18 and 19 and within a 6.6-kb region between sites 28 and 29. Microsatellite analysis revealed only one major peak at D5S346 and two peaks at D5S1965 in both KT25 and KT64 patients (data not shown), being compatible with the gene dose assay results (Fig. 3).

Determination of deleted sequences in KT25 and KT64 patients. The deletion boundary sequences were amplified by PCR with primers designed to hybridize to the region where the deletion breakpoints were located by the gene dose mapping (Fig. 4). When the DNA of KT25 was used as a template,

A

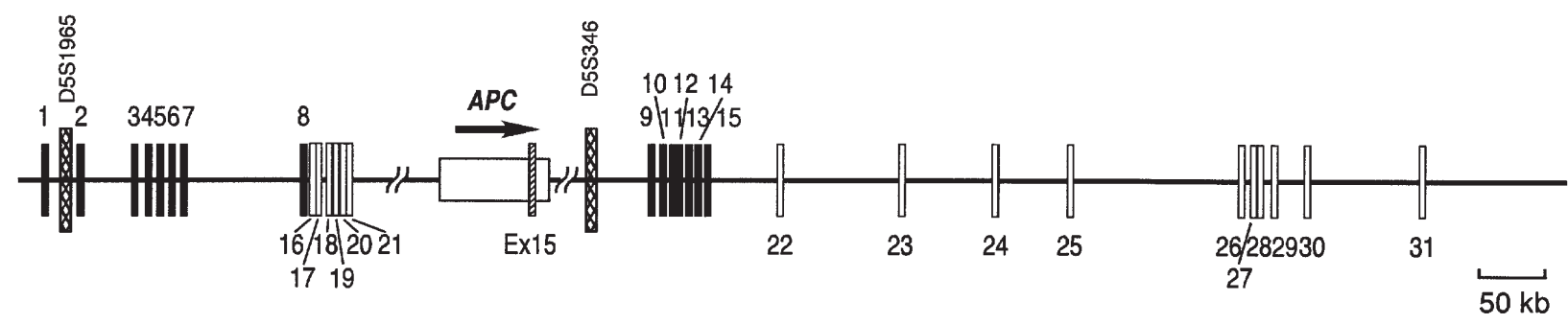

B
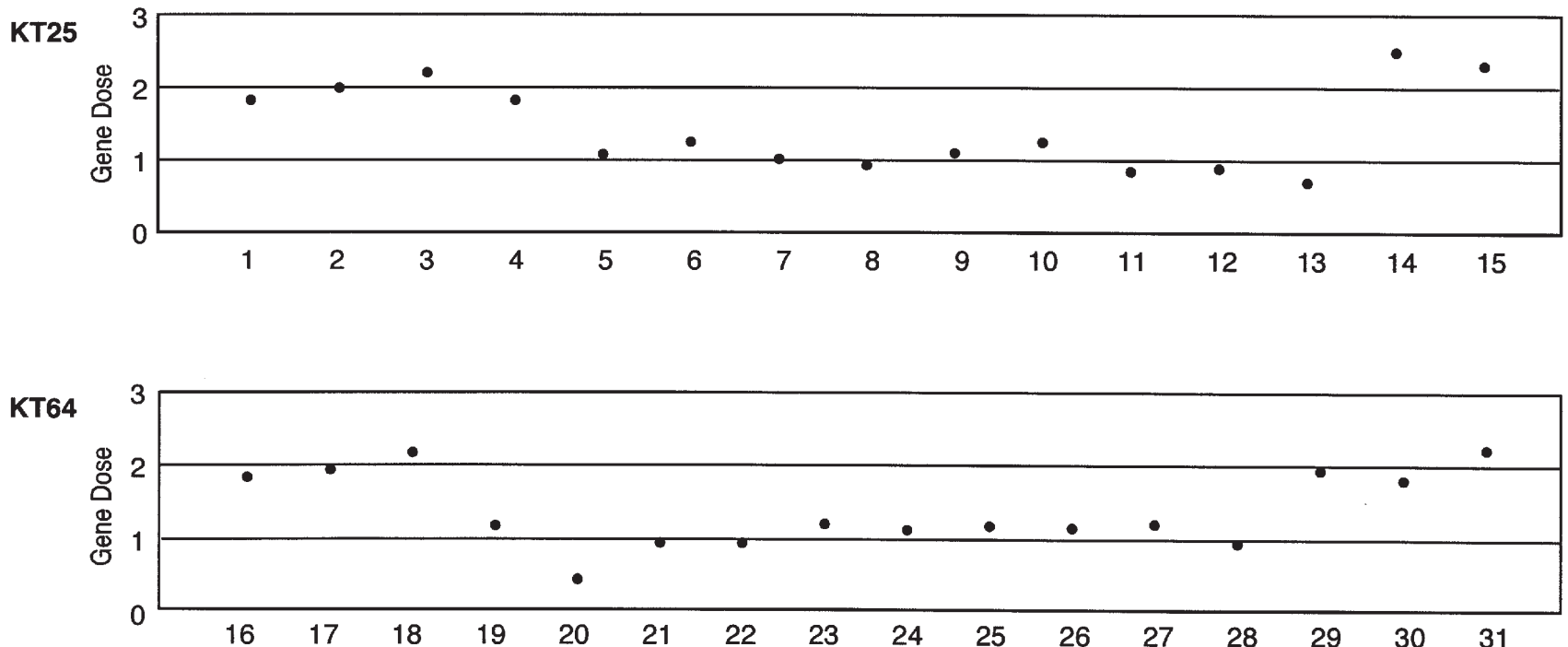

Figure 3. Gene dose mapping around the $A P C$ gene. A, schematic presentation of the genomic region around the $A P C$ gene. The closed and open bars indicate the target sites where gene dosage was measured for KT25 and KT64 patients, respectively. The hatched bar indicates the target site Ex15 within exon 15 of the gene. The open box and cross-hatched bars depict the $A P C$ gene region and microsatellite regions, respectively. The arrow indicates the transcriptional direction of the APC gene. B, gene dosage profiles of KT25 (upper) and KT64 (lower) patients. The number along the horizontal axis indicates the target site as shown in the above diagram. 

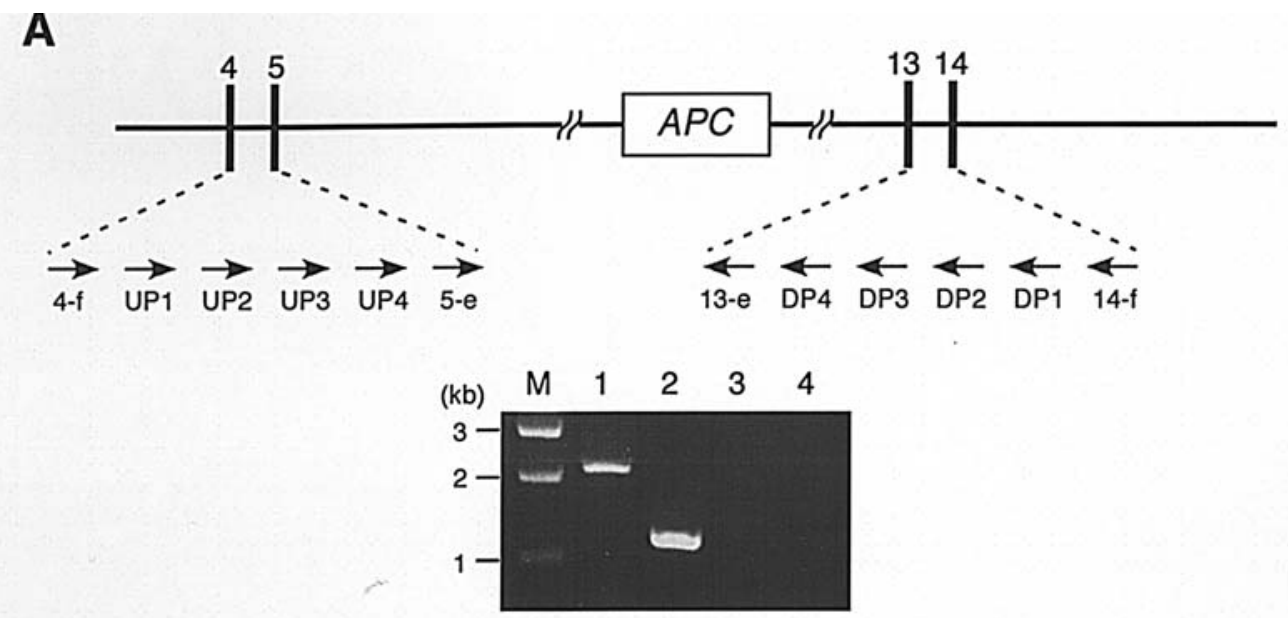

a GGATACTGGTCTTGTGTCATCACCTCCTTCCTAGTC

b : : : : : : : : : : : : : : : : : : : :ttggg: :acttgga:a

c tttg:gatt:tgc::c: : : : : : : : : : : : : : : : : : :

B

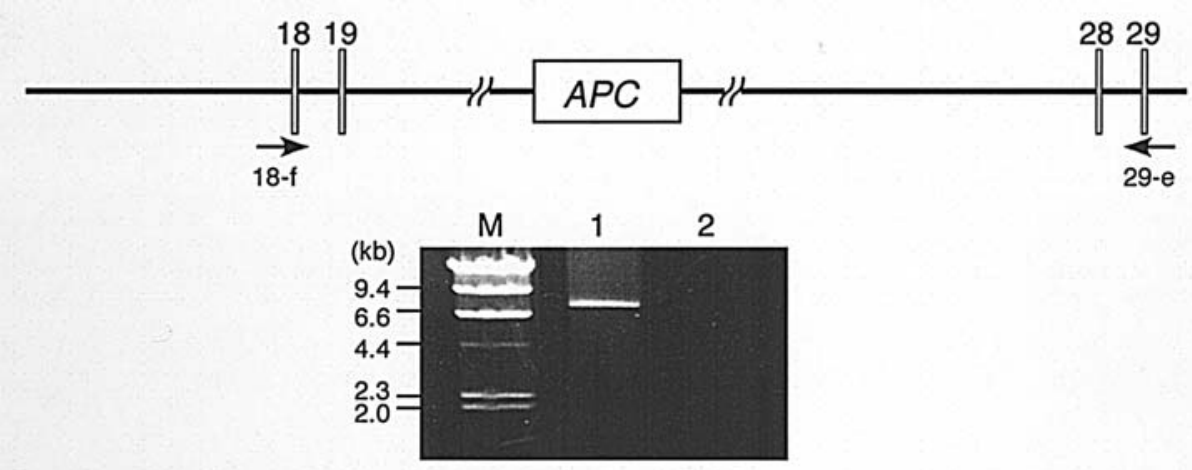

\begin{abstract}
a TTTTTTGAGGATTTCGCATCAATGTTCATCAGGGATATTGGTCTAAAATTCTCT
b : : : : : : : : : : : : : : : : : : : : : : : : : : : : : : : : : : : : : : : : : : : : : : : : : : : : : : :

C : : :a: : : : : : : : :t: : : : : : : : : : : : : :a: : : : : : : : : : : : : : : : : : : :

d : : :a: : : : : : : : : : :t: : : : :g: : : : : : : : :a: : : : : : : : : : : : : : : : : : : : :
\end{abstract}

a TTTTTTTGTTGTGTCTCTGCCCGGTTTGGTATCAAGATGATGCTGGCCTCATA

b : : : : : : : : : : : : : : : : : : : : : : a: : : : : :a: : t: : : g: : : : : : : t: : : : : : : : : :

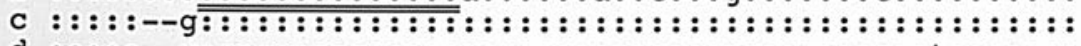

d : : : : :--g: : : : : : : : : : : : :a:c: : : :a: : : : :ga: : : : : : : :t: : : : : : :
a AAATGATTAGGGAGGATCCCTCTTTTTCTATTGATTGGAATAGTTTCAGAAGG

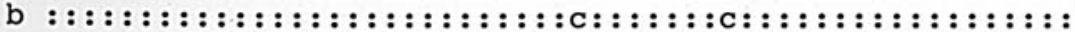

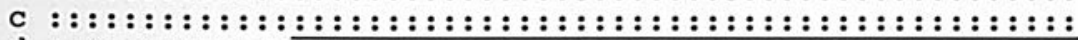

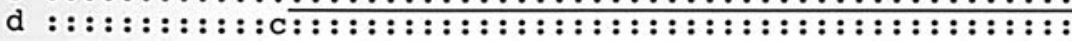
a AATGGTACCAGCTCCTCTTTGTACCTCTGGTAGAATTGGCTGTGAATCCATCTG
b $::::::::::::::$ t- $:------::::::::::::::: a::::::::::$ g: : : :
C : : : : : : : : : : : : : : : : : : : : : : : : : : : : : : : : : :c: : : : : : : : : : : :t: : : :

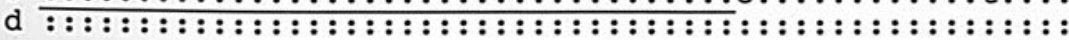

Figure 4. Molecular cloning and determination of the deletion boundaries in KT25 (A) and KT64 (B). In the upper diagrams, arrows, the open box and vertical bars indicate PCR primers, the APC gene region and target sites of gene dose assay (numbered as in Fig. 3), respectively. Ethidium bromide-stained agarose gels displaying PCR products are shown in the middle. M, DNA ladder for size marker. Nucleotide sequences of the deletion boundaries are shown below. Nucleotide sequences of the breakpoint-containing PCR products (upper case letters) and registered sequences in the database (lower case letters) are aligned where colons and hyphens indicate identical nucleotides and nucleotide gaps, respectively. A, in KT25, PCR was conducted with every possible combination between upstream and downstream primers indicated. The primers UP1-4 and DP1-4 were designed at an $\sim 1-\mathrm{kb}$ interval within regions between sites 4 and 5 and between 13 and 14. Only the primer pair of UP2 and 13-e (lane 1) and the primer pair UP3 and 13-e (lane 2) generated PCR products with KT25 DNA whereas no such products were generated with healthy control DNA (lanes 3 and 4). a, the deletion boundary sequence of the PCR product. The 4-bp sequence flanking the deletion is underlined. b, the database sequence from position 114956 (left) to 114921 (right) of AC137549.1. c, the database sequence from position 106397 (left) to 106432 (right) of AC008536.7 (right). B, in KT64, long-range PCR was conducted with primers 18-f and 29-e. Lane 1, KT64; lane 2, healthy control DNA. a, the deletion boundary sequence of the PCR product. b, the LINE-1 sequence located between 18 and 19 , from position 113107 (left upper) to 112898 (right lower) of AC136500.1. c, the LINE-1 sequence from position 43820 (left upper) to 43603 (right lower) of AC010431.7, which is located $50 \mathrm{~kb}$ further downstream from the LINE-1 element present between 28 and 29. d, the LINE-1 sequence located between 28 and 29 , from position 99225 (left upper) to 99008 (right lower) of AC010431.7. The double and single horizontal lines indicate intervals in which the recombination was suspected to occur between the LINE-1 sequences $\mathrm{b}$ and $\mathrm{c}$ and between $\mathrm{c}$ and $\mathrm{d}$, respectively. 
A

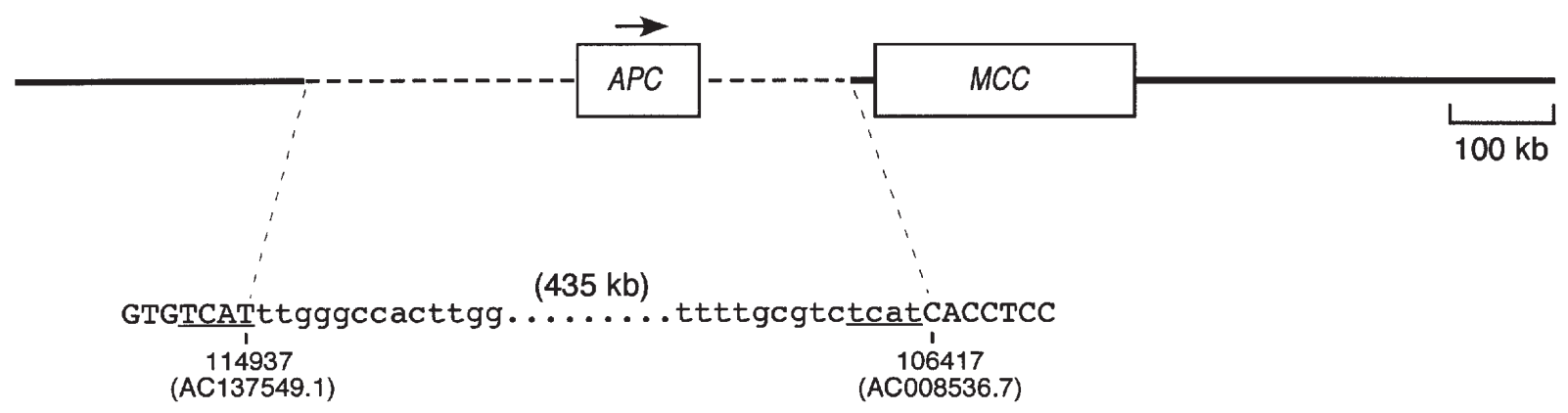

B

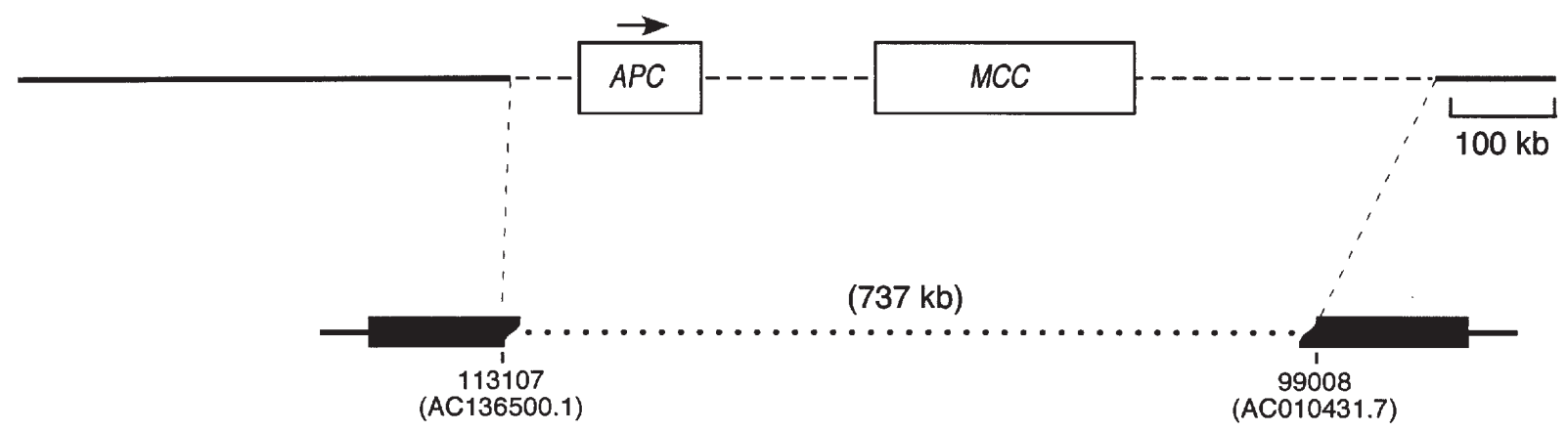

Figure 5. Schematic presentation of large deletions in KT25 (A) and KT64 (B). Solid and broken horizontal lines represent retained and deleted regions, respectively. Open boxes indicate $A P C$ and $M C C$ gene regions. The arrow indicates the direction of transcription of the $A P C$ gene. Numbers indicate nucleotide positions in the registered sequences shown in parenthesis. In A, nucleotide sequences at the deletion breakpoints are shown below. Lower case letters indicate deleted sequences. A 4-bp repeat at the breakpoint is underlined. In B, LINE-1 sequences are shown below by solid bars. The indicated nucleotide positions correspond to those shown in Fig. 4B. In place of the large deleted sequence, a short sequence of 46-139 bp, which appears to have been derived from another LINE-1 sequence, was inserted between the two half LINE-1 elements shown in this diagram.

PCR with the primer pair UP2 and 13-e and that with UP3 and 13-e generated PCR products of 2.3 and $1.3 \mathrm{~kb}$, respectively (Fig. 4A), which were not generated from the control DNA. Sequencing analysis of these PCR products confirmed that the $1.3-\mathrm{kb}$ sequence was a part of the $2.3-\mathrm{kb}$ sequence. Comparison of these sequences with those registered in the database revealed that deletion breakpoints in KT25 were located between an $\sim 224$-kb upstream site (nucleotide position 114937 numbered in AC137549.1) and an 120-kb downstream site (position 106417 in AC008536.7). The deletion was $\sim 435 \mathrm{~kb}$ in size and contained the whole $A P C$ gene (Fig. 5). There was no highly homologous sequence in the deletion boundaries except a 4-bp identical sequence at the deletion end-points.

In KT64, the regions where the breakpoints were located by gene dose mapping consisted mostly of long interspersed repetitive sequences called LINE-1 (16). Because of the high sequence homology among LINE-1 members, PCR primers for a unique sequence could not be designed within these regions. Therefore, genomic DNA was amplified by long-range PCR with the primers 18-f and 29-e (Fig. 4B). This PCR generated a 7-kb fragment from KT64 DNA but not from the control DNA (Fig. 4B). Analysis of restriction enzyme recognition sites of this fragment and comparison with database sequences revealed that the contiguous $1.2-\mathrm{kb}$ and $1.6-\mathrm{kb}$ fragments generated by KpnI digestion of the 7-kb fragment contained the deletion boundary sequences (data not shown). These KpnI fragments were introduced into a plasmid vector and analyzed by nucleotide sequencing. Comparison of these sequences with the database indicated that the boundary sequences consisted of three LINE-1 elements that were present in the same orientation around the $A P C$ gene (Fig. 4B). The upstream sequence of the boundary was approximately half $(2.5 \mathrm{~kb})$ of the LINE-1 element located $\sim 80 \mathrm{~kb}$ upstream (registered in AC136500.1) from the APC gene. The downstream sequence of the boundary was approximately half $(2.7 \mathrm{~kb})$ of the LINE-1 element located $\sim 570 \mathrm{~kb}$ downstream (registered in AC010431.7) from the APC gene. Between these two LINE-1 sequences, a short sequence of a third LINE-1 element was inserted, which seemed to be derived from the LINE-1 element located $\sim 620 \mathrm{~kb}$ downstream (registered in AC010431.7) from the APC gene. Although the size of this short insertion was estimated to be between 46 and $139 \mathrm{bp}$, the exact size and breakpoints cannot be determined because of high homology among the LINE-1 sequences. Thus, the deletion seems to have been generated by complex processes involving three LINE-1 elements. The deletion was $\sim 737 \mathrm{~kb}$ and included the whole $M C C$ gene (17) as well as the $A P C$ gene (Fig. 5).

\section{Discussion}

Genomic deletion has increasingly been recognized as a fairly common mutation of a tumor suppressor gene that should be searched for when routine PCR-based analysis fails to identify a causative mutation. The deletions vary widely in size, ranging 
from a single exon to an extremely large chromosomal region containing many genes. To detect these deletions, various techniques have been employed such as Southern blot analysis (5), fluorescence in situ hybridization (18), long-range PCR (19) and several newly developed methods for gene dosage measurement. The latter methods, which estimate the copy number of specific sequences, include competitive PCR $(12,20,21)$, multiplex PCR $(22,23)$, real-time PCR (10), multiplex amplifiable probe hybridization (24), multiplex ligation-dependent probe amplification (25) and comparative genome hybridization (26). Although various methods are thus available, no single method is adequate for every occasion and combination of these techniques is necessary in some cases. In a DNA diagnosis setting, demonstration of gene dosage alteration may not be sufficient for definite conclusion and determination of genomic breakpoints may be required.

We previously demonstrated that gene dosage assay based on competitive PCR was useful for identification of MEN1 gene deletion and determination of deletion end-points (13). In the present study, we partly modified the procedure of preparing internal standard DNA. This modification made the construction of internal standard easier, and facilitated gene dose assay at newly adopted target sites. This modified assay was applied to screening for $A P C$ gene deletion and then to determination of exact deletion breakpoints in FAP patients. Two patients were identified as a deletion carrier by the initial screening of the exon 15 copy number variation and were ultimately shown to have a $435-\mathrm{kb}$ and $737-\mathrm{kb}$ genomic deletion including the whole $A P C$ gene. Another patient was suspected to have $A P C$ gene duplication because there appeared to be three copies of exon 15. Further analysis is necessary to characterize the $A P C$ gene mutation in this patient. We found three patients having $A P C$ gene deletion or possible rearrangement among 41 patients. Similar detection rate, 7 patients with large deletion among 60 classical FAP patients, was previously reported in the screening for copy number variation of the $A P C$ exon 14 by real-time quantitative multiplex PCR assay (10).

We determined the deleted genomic sequences, which were flanked by only a 4-bp repeat in one patient and LINE-1 repetitive sequences of $\sim 6 \mathrm{~kb}$ in another patient. The involvement of short direct repeats of a few nucleotides at the deletion breakpoint has been well documented where DNA polymerase slippage has been suggested as the mechanism of generating genomic deletions $(13,27,28)$. Interspersed repetitive sequences such as LINE-1 and Alu retrotransposon elements have also repeatedly been implicated in gene rearrangements (27-29). Generation of large genomic deletions can be mediated by these elements through retrotransposition (30) or non-allelic homologous recombination (27-29). The latter mechanism is likely to be responsible for the creation of the chimeric LINE-1 element identified at the deletion breakpoint in KT64. The lack of structural hallmarks of retrotransposition such as $5^{\prime}$ truncation and a $3^{\prime}$ poly(A) tail is also consistent with the homologous recombination mechanism. However, the presence of a third LINE-1 sequence of 46-139 bp in the chimeric element at the breakpoint suggests that the recombination was not a simple crossover event between two LINE-1 elements. Similar three-piece chimeras have been reported for Alu elements associated with large genomic deletions, where $\sim 130$-bp Alu repeat sequences were inserted $(27,28)$. Although a model has been proposed for its formation process assuming the contribution of an Okazaki fragment from a remote repetitive element $(27,28)$, the precise mechanism for the creation of a three-piece chimera associated with a large genomic deletion remains to be elucidated.

The delineation of genomic breakpoints in affected families will provide a much stronger proof of gene deletion than reduced gene dosage or reduced fluorescence in situ hybridization signals alone, and consequently offer important information for genetic counseling of the patients' families. Our gene dosage mapping successfully delimited the deletion boundaries and led to the determination of breakpoints only with a small amount of clinical DNA samples that remained after the initial mutation screening. Thus, the gene dose mapping has proved to be a useful technique for a definitive DNA test of large gene deletions in a clinical setting.

\section{Acknowledgements}

This study was supported in part by the program for promotion of Fundamental Studies in Health Sciences of the Pharmaceuticals and Medical Devices Agency, by Grant-in-aid for Scientific Research from Japan Society for the Promotion of Science and by SRF for Biomedical Research. M.T. is an Awardee of Research Resident Fellowship from the Foundation for Promotion of Cancer Research (Japan) for the 3rd Term Comprehensive 10-Year-Strategy for Cancer Control.

\section{References}

1. Lynch HT and De la Chapelle A: Genetic susceptibility to nonpolyposis colorectal cancer. J Med Genet 36: 801-818, 1999.

2. Groden J, Thliveris A, Samowitz W, et al: Identification and characterization of the familial adenomatous polyposis coli gene. Cell 66: 589-600, 1991.

3. Kinzler KW, Nilbert MC, Su L-K, et al: Identification of FAP locus genes from chromosome 5q21. Science 253: 661-665, 1991.

4. Miyoshi Y, Ando H, Nagase $\mathrm{H}$, et al: Germ-line mutations of the $A P C$ gene in 53 familial adenomatous polyposis patients. Proc Natl Acad Sci USA 89: 4452-4456, 1992.

5. Van der Luijt RB, Khan PM, Vasen HFA, et al: Molecular analysis of the $A P C$ gene in 105 Dutch kindreds with familial adenomatous polyposis: 67 germline mutations identified by DGGE, PTT, and Southern analysis. Hum Mutat 9: 7-16, 1997.

6. Heinimann K, Thompson A, Locher A, et al: Nontruncating $A P C$ germ-line mutations and mismatch repair deficiency play a minor role in $A P C$ mutation-negative polyposis. Cancer Res 61: 7616-7622, 2001.

7. Armstrong JG, Davies DR, Guy SP, Frayling IM and Evans DGR: $A P C$ mutations in familial adenomatous polyposis families in the Northwest of England. Hum Mutat 10: 376-380, 1997.

8. Gavert N, Yaron Y, Naiman T, et al: Molecular analysis of the $A P C$ gene in 71 Israeli families: 17 novel mutations. Hum Mutat 19: 664, 2002.

9. Bisgaard ML, Ripa RS and Bülow S: Mutation analysis of the adenomatous polyposis coli $(A P C)$ gene in Danish patients with familial adenomatous polyposis (FAP). Hum Mutat 23: 522, 2004.

10. Sieber OM, Lamlum H, Crabtree MD, et al: Whole-gene APC deletions cause classical familial adenomatous polyposis, but not attenuated polyposis or 'multiple' colorectal adenomas. Proc Natl Acad Sci USA 99: 2954-2958, 2002.

11. Armour JAL, Barton DE, Cockburn DJ and Taylor GR: The detection of large deletions or duplications in genomic DNA. Hum Mutat 20: 325-337, 2002.

12. Kishi M, Tsukada T, Shimizu S, et al: A large germline deletion of the MEN1 gene in a family with multiple endocrine neoplasia type 1. Jpn J Cancer Res 89: 1-5, 1998. 
13. Kikuchi M, Ohkura N, Yamaguchi K, Obara T and Tsukada T: Gene dose mapping delineated boundaries of a large germline deletion responsible for multiple endocrine neoplasia type 1. Cancer Lett 208: 81-88, 2004.

14. Prosser J, Condie A, Wright M, Horn JM, Fantes JA, Wyllie AH and Dunlop MG: $A P C$ mutation analysis by chemical cleavage of mismatch and a protein truncation assay in familial adenomatous polyposis. Br J Cancer 70: 841-846, 1994.

15. Yamakita N, Murai T, Ito Y, et al: Adrenocorticotropinindependent macronodular adrenocortical hyperplasia associated with multiple colon adenomas/carcinomas which showed a point mutation in the APC gene. Intern Med 36: 536-542, 1997.

16. Burton FH, Loeb DD, Voliva CF, Martin SL, Edgell MH and Hutchison CA III: Conservation throughout mammalia and extensive protein-encoding capacity of the highly repeated DNA long interspersed sequence one. J Mol Biol 187: 291-304, 1986.

17. Kinzler KW, Nilbert MC, Vogelstein B, et al: Identification of a gene located at chromosome 5q21 that is mutated in colorectal cancers. Science 251: 1366-1370, 1991.

18. Pack SD, Zbar B, Pak E, et al: Constitutional von HippelLindau $(V H L)$ gene deletions detected in VHL families by fluorescence in situ hybridization. Cancer Res 59: 5560-5564, 1999.

19. Payne SR, Newman B and King M-C: Complex germline rearrangement of BRCA1 associated with breast and ovarian cancer. Genes Chromosomes Cancer 29: 58-62, 2000.

20. Deng G, Yu M and Smith HS: An improved method of competitive PCR for quantitation of gene copy number. Nucleic Acids Res; 21: 4848-4849, 1993.

21. Celi FS, Cohen MM, Antonarakis SE, Wertheimer E, Roth J and Shuldiner AR: Determination of gene dosage by a quantitative adaptation of the polymerase chain reaction (gd-PCR): rapid detection of deletions and duplications of gene sequences. Genomics 21: 304-310, 1994.
22. Charbonnier F, Raux G, Wang Q, et al: Detection of exon deletions and duplications of the mismatch repair genes in hereditary nonpolyposis colorectal cancer families using multiplex polymerase chain reaction of short fluorescent fragments. Cancer Res 60: 2760-2763, 2000.

23. Wang Y, Friedl W, Sengteller M, Jungck M, Filges I, Propping P and Mangold E: A modified multiplex PCR assay for detection of large deletions in MSH2 and MLH1. Hum Mutat 19: 279-286, 2002.

24. Armour JAL, Sismani C, Patsalis PC and Cross G: Measurement of locus copy number by hybridisation with amplifiable probes. Nucleic Acids Res 28: 605-609, 2000.

25. Gille JJP, Hogervorst FBL, Pals G, et al: Genomic deletions of MSH2 and MLH1 in colorectal cancer families detected by a novel mutation detection approach. Br J Cancer 87: 892-897, 2002.

26. Bruder CEG, Hirvelä C, Tapia-Paez I, et al: High resolution deletion analysis of constitutional DNA from neurofibromatosis type 2 (NF2) patients using microarray-CGH. Hum Mol Genet 10: 271-282, 2001.

27. Nicholls RD, Fischel-Ghodsian N and Higgs DR: Recombination at the human $\alpha$-globin gene cluster: sequence features and topological constraints. Cell 49: 369-378, 1987.

28. Kutsche K, Ressler B, Katzera H-G, et al: Characterization of breakpoint sequences of five rearrangements in L1CAM and $A B C D 1$ ( $A L D$ ) genes. Hum Mutat 19: 526-535, 2002.

29. Burwinkel B and Kilimann MW: Unequal homologous recombination between LINE-1 elements as a mutational mechanism in human genetic disease. J Mol Biol 277: 513-517, 1998.

30. Gilbert N, Lutz-Prigge S and Moran JV: Genomic deletions created upon LINE-1 retrotransposition. Cell 110: 315-325, 2002. 